\title{
Balloon-Distension Studies in the Gastrointestinal Tract: Current Role
}

\author{
Hans Gregersen ${ }^{a-c}$ Asbjørn M. Drewes ${ }^{a, b}$ Barry P. McMahon ${ }^{a, d}$ \\ Donghua Liao ${ }^{a}$ \\ ${ }^{a}$ Center of Excellence in Visceral Biomechanics and Pain, Aalborg Hospital, Aalborg, and ${ }^{b}$ Institute of

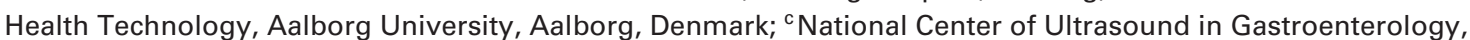 \\ Bergen University and Haukeland Hospital, Bergen, Norway; ${ }^{\mathrm{d}}$ Department of Medical Physics and \\ Clinical Engineering, $\mathrm{AMNCH}$, Dublin, Ireland
}

\section{Key Words}

Balloon distension $\cdot$ Impedance planimetry $\cdot$ Muscle

function - Tension - Cross-section area loon-distension techniques, in particular new developments of the impedance planimetric technique including methods for studying gastrointestinal muscle function.

Copyright (C) 2006 S. Karger AG, Basel

\section{Introduction}

Balloon (bag) distension is a commonly used technique in visceral organs. Research studies take advantage of this technique for studying organ physiology, e.g. for investigating the force-deformation relationship and mechanosensitive receptors in the gastrointestinal (GI) wall. Balloon distension is also used for diagnostic purposes, e.g. in the experimental diagnosis of non-cardiac chest pain [1] and for treatment of diseases such as atherosclerosis [2], bleeding esophageal varices caused by liver disease and lower esophageal sphincter occlusion caused by achalasia [3].

Distension techniques can be used in vivo and in vitro with concomitant measurements of pressure, volume and cross-sectional area (CSA) individually or in combination. Furthermore, balloon distension can be done in conjunction with imaging techniques such as B-mode ultra-

\section{KARGER}

Fax +4161306 1234 E-Mail karger@karger.ch www.karger.com
Hans Gregersen, MD, DrMSc

Center for Visceral Biomechanics and Pain

The Research Building, Aalborg Hospital

Søndre Skovvej 15, DK-9000 Aalborg (Denmark)

Tel. +459932 6825, Fax +459932 6801, E-Mail hag@miba.auc.dk 
sonography and MRI to obtain geometric data about three-dimensional (3D) surfaces for further calculation of tension and stress in GI organs such as the stomach $[4,5]$ and rectum [6].

Balloon-distension techniques that are purely based on simultaneous pressure-volume measurements do not have a sound basis in the physical sciences and are therefore not a good platform to base comparative studies between individual responses to balloon distension. The measurements of pressure and volume may be accurate but any derived parameter such as tension is invalid due to the numerous geometric assumptions made and the errors caused by elongation and contractions. Errors in the biomechanical analysis have consequences for interpretation of data on tone, tension, and distensibility [7]. Local geometric information, for example the cross-section area for the tubular organ and the 3D radii of curvature of the irregular complex organs, is important. Thus, advanced distension technology is needed.

This article describes balloon techniques including barostat and impedance planimetry. Furthermore, several methods based on impedance planimetry including novel analysis of smooth muscle function are introduced.

\section{Simple Balloon Techniques}

Most balloon distensions are done with simultaneous measurement of the balloon pressure and volume. This can be done with fluid or air-filled balloons. An example of the latter is the barostat technique. The barostat was designed as a device to record GI motility and for evaluation of tone in the gastric fundus. It has been used in numerous studies. The basic principle of the barostat is to maintain a constant pressure within the balloon/bag in the lumen even though muscular contractions and relaxations occur $[8,9]$. To maintain a constant pressure, the barostat aspirates air into the balloon during contractions and injects air during relaxation. The great advantage of the barostat system and similar pressure-volume-based methods is the relatively low cost if the system is customor home-made by the user, and the barostat is easy to use for routine purposes. It has been used for determination of sensory and pain thresholds and under different conditions attempts have been done to calculate the compliance and tension of the organs [8, 10-12].

With respect to calculation of mechanical factors, balloon-distension methods need to be considered in greater detail. In general, the boundary of a geometrically mea- surable structure must be in complete contact with the measurand, i.e. when measuring the volume of an organ, the whole surface area of the balloon must be in contact with the tissue. It is important to ensure that the applied pressure is properly transmitted to the tissue, i.e. that the balloon is sufficiently large so that it does not contribute its own elastic properties to the results (therefore, the word balloon is in a way misleading - bag is a more appropriate word). Nowadays, many distension studies use a polyethylene bag rather than a latex balloon since latex balloons resist inflation and thus show a rapid increase in intra-balloon pressure with small volumes of distension $[8,13,14]$.

The issue discussed above has implications for the measurement of muscle tone. However, there are other difficulties. The definition of specific tone (muscle tone not associated with phasic contractility) does not apply for studies based on pressure-volume data since this technique provides a 3D measure of tone that is difficult to differentiate from phasic contractility (tetanic tone is a summation of phasic contractions). The dynamic characteristics of the measurement system also make it difficult to compare results from different studies, e.g. short balloons encounter only a few contractions whereas long balloons sum up phasic contractions in a tetanic tone pattern. The biomechanical analyses in pressure-volume studies seldom take into consideration the proper geometry and thus assume that the tubular sections of the GI tract are circular and that the stomach is spherical. Due to these limitations with balloon techniques, it is required that measurements are obtained at separate axial locations in the tubular parts of the GI tract, i.e. the luminal CSA and the 3D surface analysis for the complex organ. The current knowledge therefore is based on qualitative data and more sophisticated analysis is greatly needed.

\section{Impedance Planimetry}

Impedance planimetry is a technique for measurement of luminal CSA in hollow organs, especially in cylindrical tubes (fig. 1). It has been used to measure CSA together with bag pressure in animal and human studies and the wall tension and strain in the organ can be estimated $[15,16]$.

From Ohm's law we know that V = IZ, where V, I and $\mathrm{Z}$ denote the potential difference, the current and the electrical impedance. In the case of a fluid-filled bag on a probe with an alternating constant current set up between two excitation electrodes (E), Ohm's law will apply 
for the field between two inner sensing electrodes (S). The impedance depends on the distance (D) between the $\mathrm{S}$ electrodes, the conductivity $(\sigma)$ of the liquid in the bag and the CSA of the bag between the S electrodes. In effect

$$
\mathrm{Z}=\frac{\mathrm{D}}{\sigma \mathrm{CSA}}
$$

and therefore

$$
\mathrm{V}=\frac{\mathrm{ID}}{\sigma \mathrm{CSA}} \text {. }
$$

If the conductivity of the solution remains constant (the temperature must be constant), a calibration constant is calculated, the distance between the $\mathrm{S}$ electrodes is fixed, and the current source exciting the E electrodes is kept constant, then

$$
\mathrm{V}=\alpha \frac{1}{\mathrm{CSA}}
$$

where $\alpha$ is a calibration constant. Thus, the voltage crossing the $\mathrm{S}$ electrodes is proportional to the inverse of the CSA. Direct proportionality can be obtained by reciprocation. The data is conditioned and stored on a PC for further analysis. The electrical field created by the current from the excitation electrodes is confined to the conducting fluid inside a balloon embracing the electrodes on the probe. To control the pressure or volume inside the balloon, a channel connects the balloon with a fluid container that can be raised to increase the balloon pressure or to a pump (fig. 1).

The four-electrode system can be extended to include many sets of detection electrodes (multi-electrode systems) so that serial cross sections can be obtained on the longitudinal axis (fig. 2). This has the advantage that spatial variations in CSA can be evaluated since a third dimension is added. Thus, the spatial and temporal distribution of contractions can be described in more detail.

Newer balloon-distension methods based on impedance planimetry allows recording the luminal CSA directly and calculating the radius in the distended segment of the esophagus or intestine [17-22]. From the radius it is possible to estimate the circumferential wall tension and strain with more accuracy than from volume measurements [23]. Circularity must be assumed but has often been confirmed [20]. Finally, mechanical distension combined with ultrasonographic methods offer the possibility for a better anatomical characterization and computation of stress in the GI tract [16].

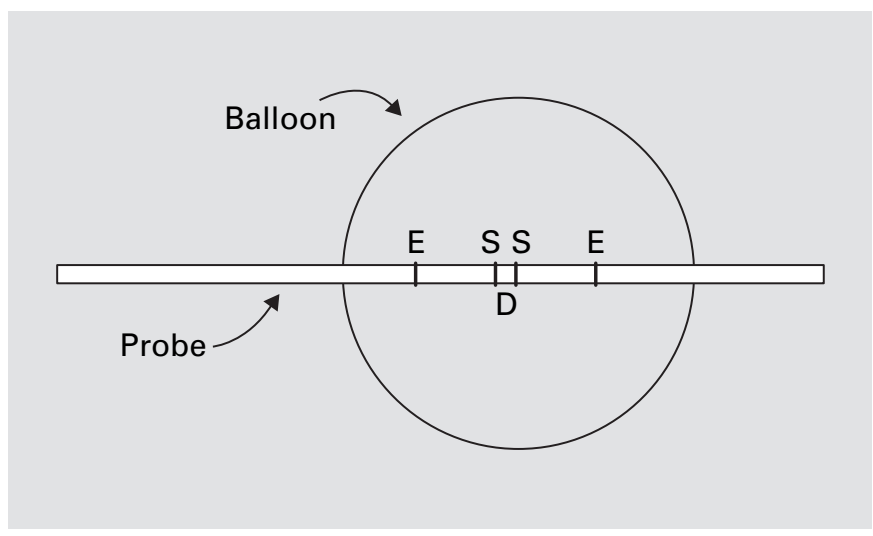

Fig. 1. Sketch of a conventional impedance planimetric probe with one set of sensing electrodes (S) for measurement of CSA in the middle of the balloon. $\mathrm{E}=$ Excitation electrodes, $\mathrm{D}=$ distance between sensing electrodes.

\section{Functional Lumen Imaging Probe}

The impedance planimetry technique has been used extensively to determine the luminal CSA in hollow organs of the body and in particular in the GI organs [20, 21, 24-29]. Impedance planimetry has recently been further developed into multi-electrode systems for the purpose of measuring a number of cross sections inside a saline-filled bag [30] in the esophagogastric junction [31]. Moreover, an impedance planimetry functional lumen imaging probe (FLIP) constructed to measure 8 CSAs has been developed (fig. 2).

The validation test of the FLIP by using a diagnostic ultrasound probe indicated in a phantom of the sphincter geometry that the mean values for CSAs in the phantom as measured with the FLIP correlate well with the mean values as measured with the ultrasound probe (fig. 3).

Until now, the FLIP is the first technique to give a clear 3D outline of the geometric profile of the sphincter at various levels of balloon distension [McMahon et al., unpubl. data] (fig. 4).

Competence of the sphincter is better derived from geometry and pressure data gathered during distension than by measuring the force at fixed points and diameters as in the case of manometry. The closely spaced CSA measurements allow the reconstruction of the geometry and this in turn allows the generation of images which represent this part of the lumen of the GI tract when it is physiologically active. The accepted understanding of esophagogastric function is that it closes off to stop the refluxing of stomach contents into the esophagus. This 


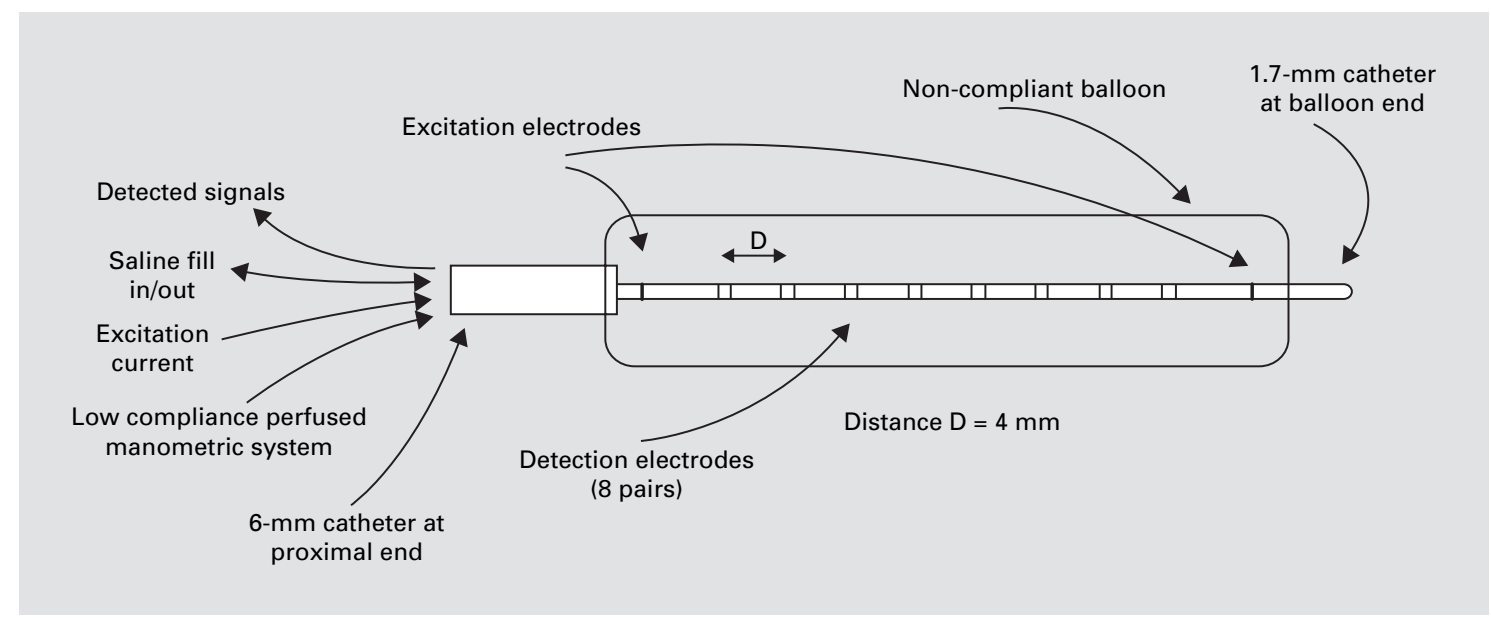

Fig. 2. Distal end of the 8 CSA probe showing electrode arrangement inside the bag-like balloon. The signals, channels, fill and perfusion channels are shown at the proximal end.

Fig. 3. Plot of radii calculated from ultrasound pull through the phantom displayed as solid line graph. Maximum and minimum values $(n=5)$ shown as dotted lines. Radii values for the 8 CSAs shown for the FLIP probe. CSA1 is most distal and CSA 8 is most proximal. The thick bar is the mean value $(\mathrm{n}=10)$ and the thin bars above and below represent the maximum and minimum measured values.

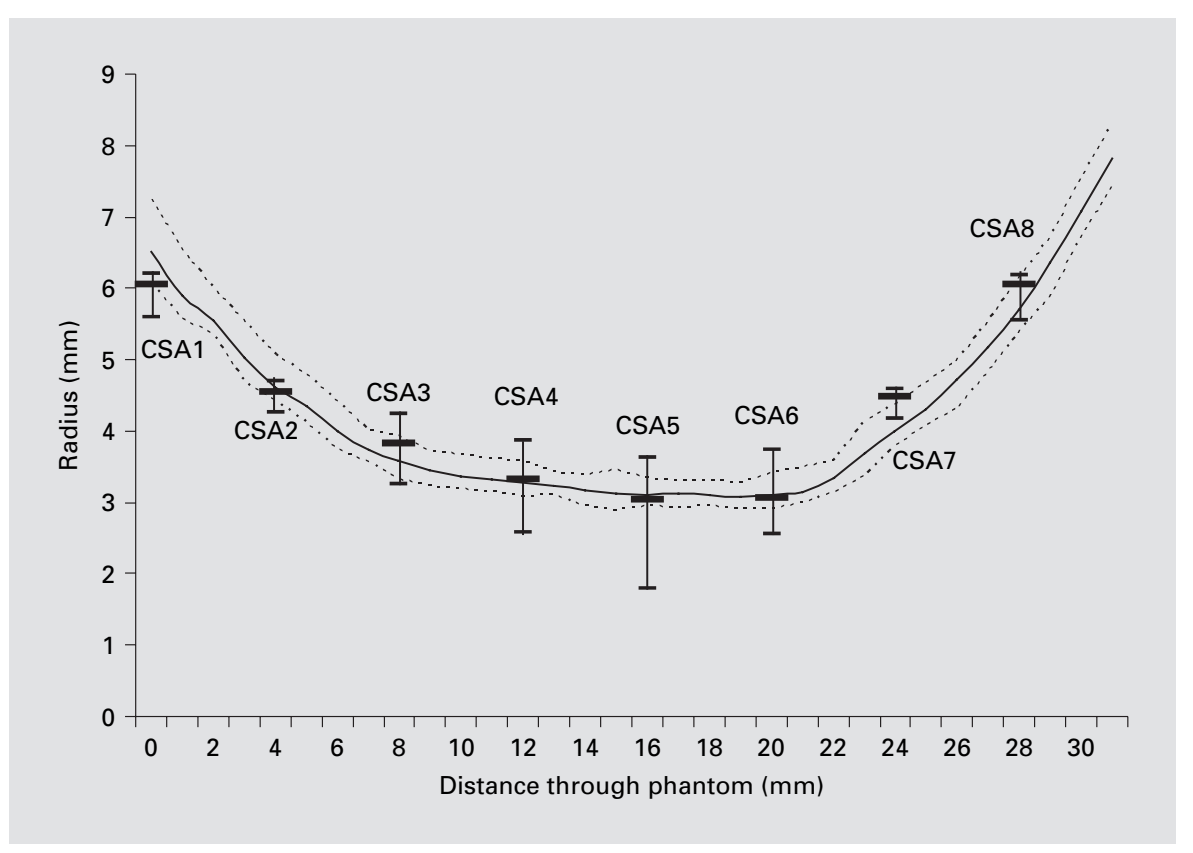

closing off must be related to a narrowing of the lumen which can be represented by CSAs measured in this region. Providing a relatively simple technique to measure this in the human body, as the technique described here does, could represent a major step forward in the diagnosis, monitoring and evaluation of diseases such as gastroesophageal reflux disease and achalasia.

Using CSA and pressure measurements the FLIP has the potential to provide new information on the biome- chanical functioning of the esophagogastric junction in healthy volunteers and in patients with disorders such as gastroesophageal reflux disease were an ineffective junction allows acid from the stomach to travel back up into the esophagus. The technique may have a role in assisting with drug therapy and surgical therapies for reflux. The technique can readily be modified for measurements in other sphincteric regions. 


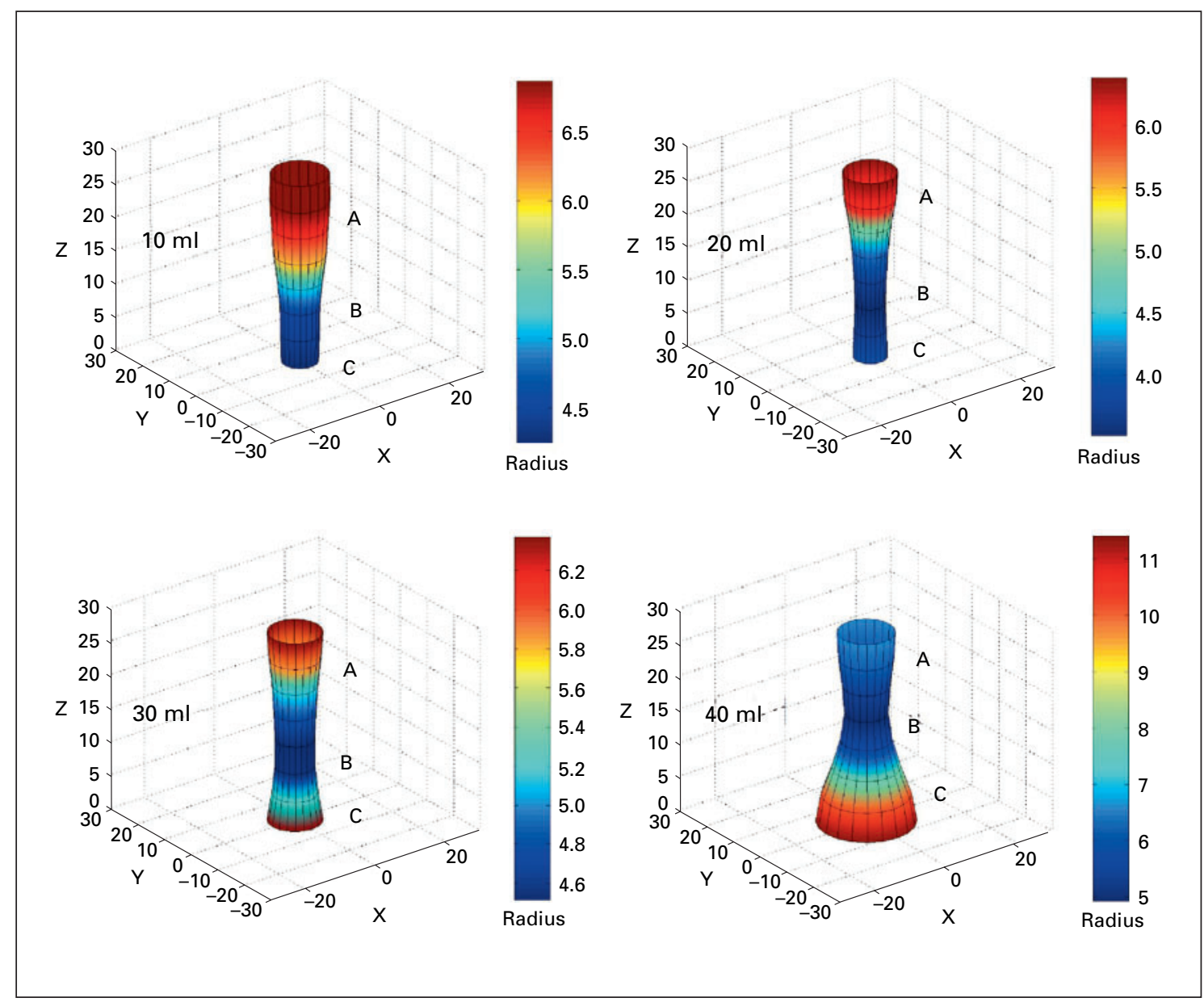

Fig. 4. 3D reconstruction of the geometry of the esophagogastric junction shown at bag fill volumes of 10, 20, 30 and $40 \mathrm{ml}$ respectively. The color change represents radii $(\mathrm{mm})$ changes. In each case, A represents the position in the luminal image of the ampulla of the esophagus, B represents the esophagogastric junction, and $\mathrm{C}$ represents the point at which the esophagus enters the stomach. $\mathrm{X}, \mathrm{Y}$ and $\mathrm{Z}$ are the $3 \mathrm{D}$ distances $(\mathrm{mm})$.

\section{New Muscle Function Analysis}

Balloon distension has become popular as a research tool for GI studies, primarily because mechanical data can be obtained. However, only few distension studies using inhibitory drugs of muscle function focused on separating phasic and tonic contractions [32, 33]. Recently, tools were developed for studying both the active (phasic and tonic contractions) and passive behavior using impedance planimetric distension before and during administration of inhibitory drugs [16, 26, 28, 29]. Tension, stress and strain are of principal interest because these parameters give information about the GI tract elastic properties and mechanoreceptors respond more directly to these parameters than to pressure and volume [34].
The muscle function is better evaluated when the forces and tensions can be measured rather than the pressure generated by contractions [34, 35].

Most mechanical studies in vitro on GI smooth muscle are isometric length-tension experiments conducted in small organ baths and during stimulation or inhibition with pharmacological substances [36]. Such studies have given valuable information about the regulation of contractions and passive tissue properties. One of the best known relationships of muscle action is the hyperbolic force-velocity relation [37, 38]. Although this relationship was originally derived for isolated muscle, it has been confirmed for whole striated muscle movements [39]. The curve implies that the velocity of muscle contraction is inversely proportional to the load, that a large force 
Fig. 5. Example of the pressure and CSA curves recorded as a function of time from the balloon distension on the human duodenum. The curves from time 100 to $150 \mathrm{~s}$ are magnified at the up left corner of the figure.

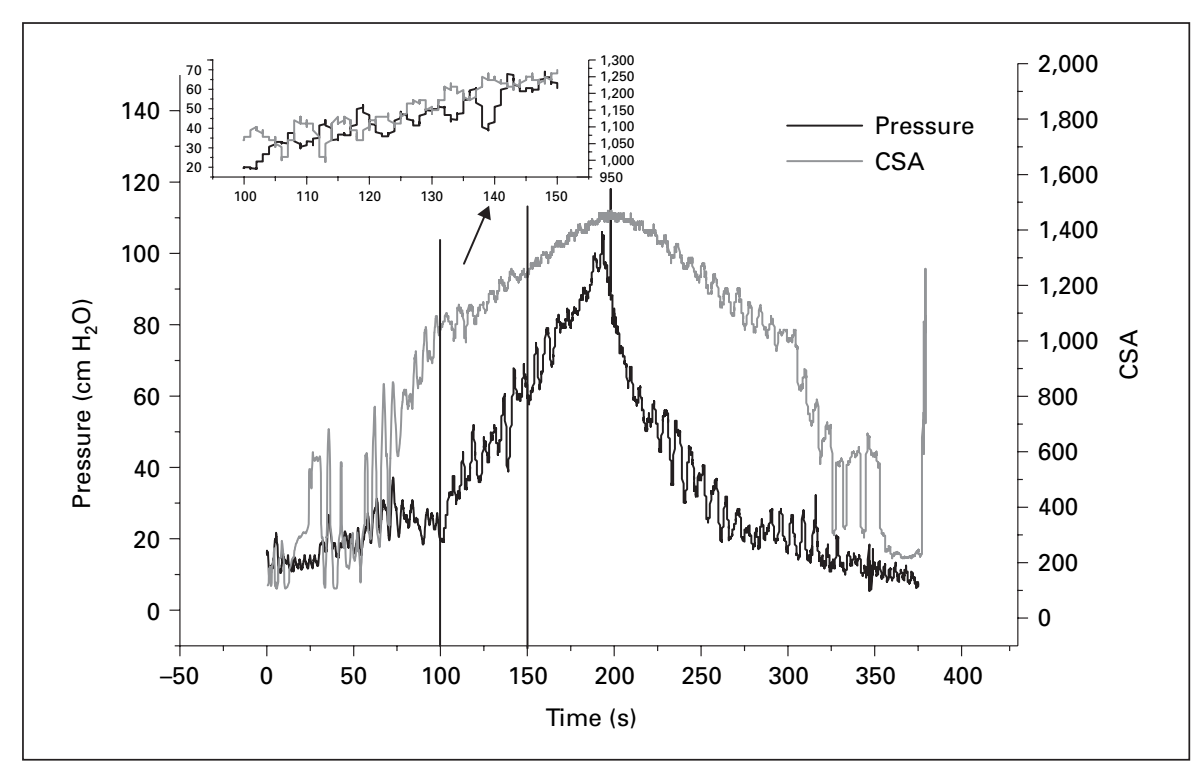

cannot be exerted in very rapid movements, that the greatest velocities are attained under conditions of low loading, and that the intermediate values of force and velocity depend on the maximal isometric force. Similar force-velocity relationships have now been described in the GI tract (see below).

GI balloon-distension studies are normally done using different step- or volume-controlled ramp protocols. A typical example of CSA and pressure recordings is shown in figure 5 where a volume-controlled ramp distension was done in the human duodenum.

Based on the basic data (CSA and pressure) obtained from balloon distension, the following analysis of the muscle function can be done:

(1) In vivo length-tension diagrams: Length-tension diagrams can be obtained in vivo in the GI tract using impedance planimetry. Passive curves obtained during administration of butylscopolamine and active curves in terms of both phasic and tonic curves can be generated by analyzing data in accordance with the tracing in figure 6. The length-tension properties can be expressed in terms of tension-stretch ratio diagrams with tension decomposed into passive, tonic and combined phasic-tonic tension. The stretch ratio was calculated from the measured CSA at the non-distension and a distension state.

(2) Force-velocity analysis: Force-velocity relationship represents some of the contraction capabilities of the muscle. Based on the in vivo balloon-distension test, the force-velocity curves can be obtained from single contractions illustrated in the pressure and CSA curves. Figure 7 shows the bag radius (derived from the CSA) and the pres- sure during ramp distension of the duodenum. The slope of the contraction curve (arrows representing the contraction velocity) decrease as a function of the degree of distension.

Force-velocity and power curves expressed as circumferential preload tension-radius shortening velocity and circumferential preload tension*CSA rate-tension diagrams are shown in figure 7 for the duodenum in controls and systemic sclerosis patients. It is clear from these diagrams that patients with systemic sclerosis have impaired muscle function. This represents the first data with application of muscle force-velocity relations in patients with GI diseases. Decreased muscle function may explain the GI symptoms reported in this patient group and should be considered as an important factor in the diagnosis and treatment.

The new analyses will widen the muscle function studies on the GI tract and the muscle disability caused by diseases can be estimated quantitatively.

\section{Multimodal Model}

As pain is a multidimensional perception it is obvious that the reaction to a single stimulus of a given modality can represent only a limited fraction of the entire pain experience. The possibility for combining different methods for stimulation and assessment will approximate the clinical situation where many different nerves and pathways are activated. Thus, the method will give a more comprehensive and differentiated information about the 
Fig. 6. A Illustration of a ramp-distension curve in the human duodenum. The open symbols above the curve mark the phasic contractions and the afterload pressure. The closed symbols under the curve mark the tonic part of the distension curve and the preload pressure. The passive pressure is measured from the tonic part of the curve during administration of butylscopolamine. B Pressure and CSA was measured and stretch ratio and tension was computed from these values.

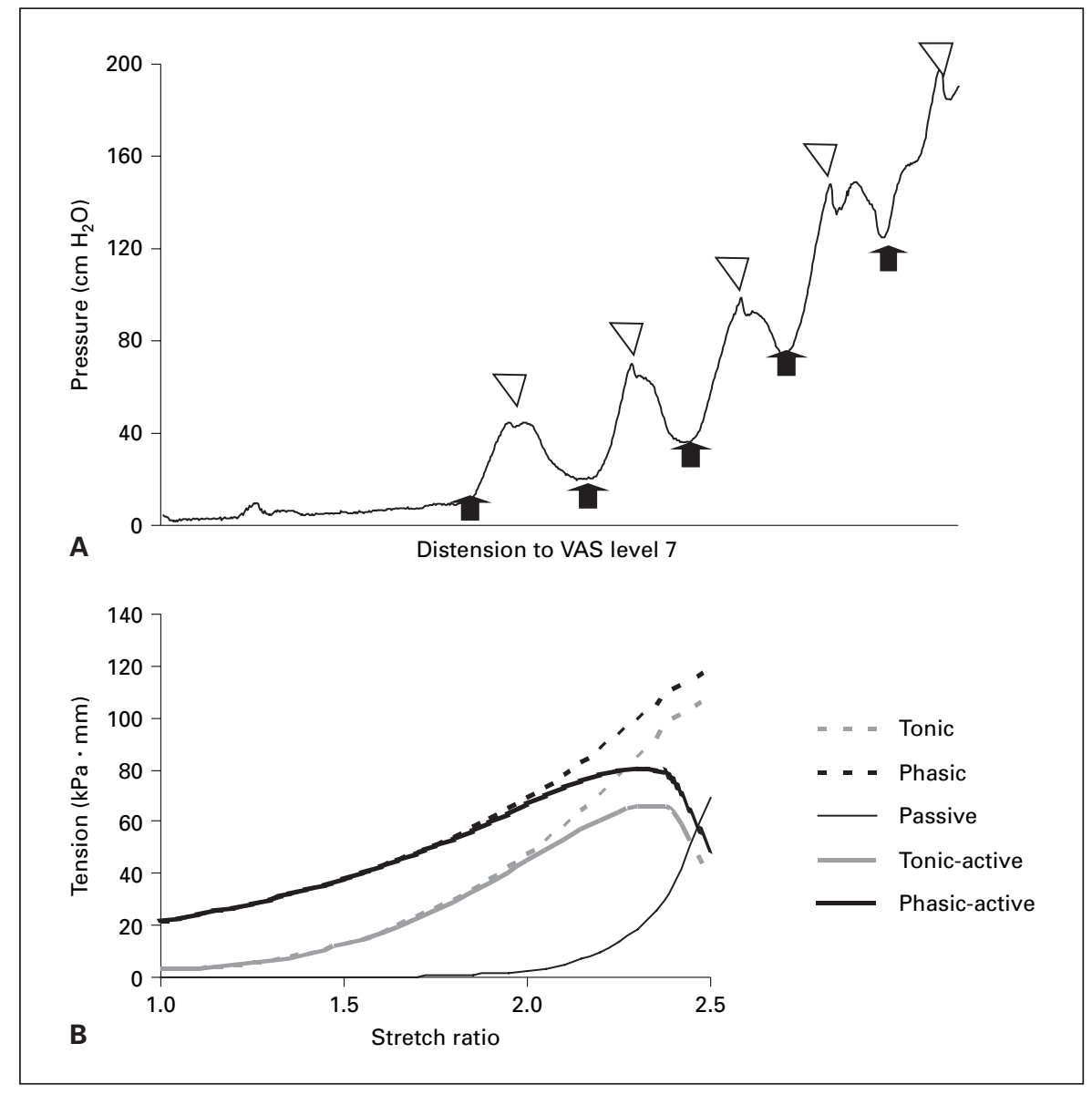

nociceptive system compared to stimuli using single or a few modalities [40]. For comprehensive experimental studies mimicking the clinical situation, a multimodal testing approach must therefore be used where the test battery will increase the probability for activation of a range of relevant nervous mechanisms. Such sophisticated methods will be able to select the best test procedures to explore different basic aspects of pain as well as pharmacological modulations. Multimodal models have shown their value in somatic pain models, where single modality models have been inadequate to test for example pathophysiological changes and effects of specific drugs [40]. Especially if the stimulation includes modalities known to evoke peripheral as well as central sensitization, the likelihood that a model will mimic clinical pain is high despite the non-harmful nature of the stimulation [24]. In somatic models it is possible to give many different stimuli and evoke central phenomena (e.g. central amplification of summated stimuli) of clinical relevance. In the GI tract, however, difficulties with access to the organs and technical limitations of the currently available models have until now made such a multimodal stimulation approach difficult. We recently introduced several multimodal models with combined chemical, mechanical, electrical, cold and warmth stimuli to assess the sensation of the esophagus together with acid sensitization [24]. The model has currently been extended to stimulate the proximal esophagus, duodenum and rectum and have been used in to unravel the pain mechanisms in different patients and in drug research (fig. 8). The multimodal approach gives the possibility for a differentiated stimulation of receptors in the superficial and deep layers of the gut.

\section{D Ultrasound Antrum Study}

Biomechanical analysis for complex geometric organs as the stomach can be based on the 3D surface geometry such as the distribution of radii of the curvature. With the 

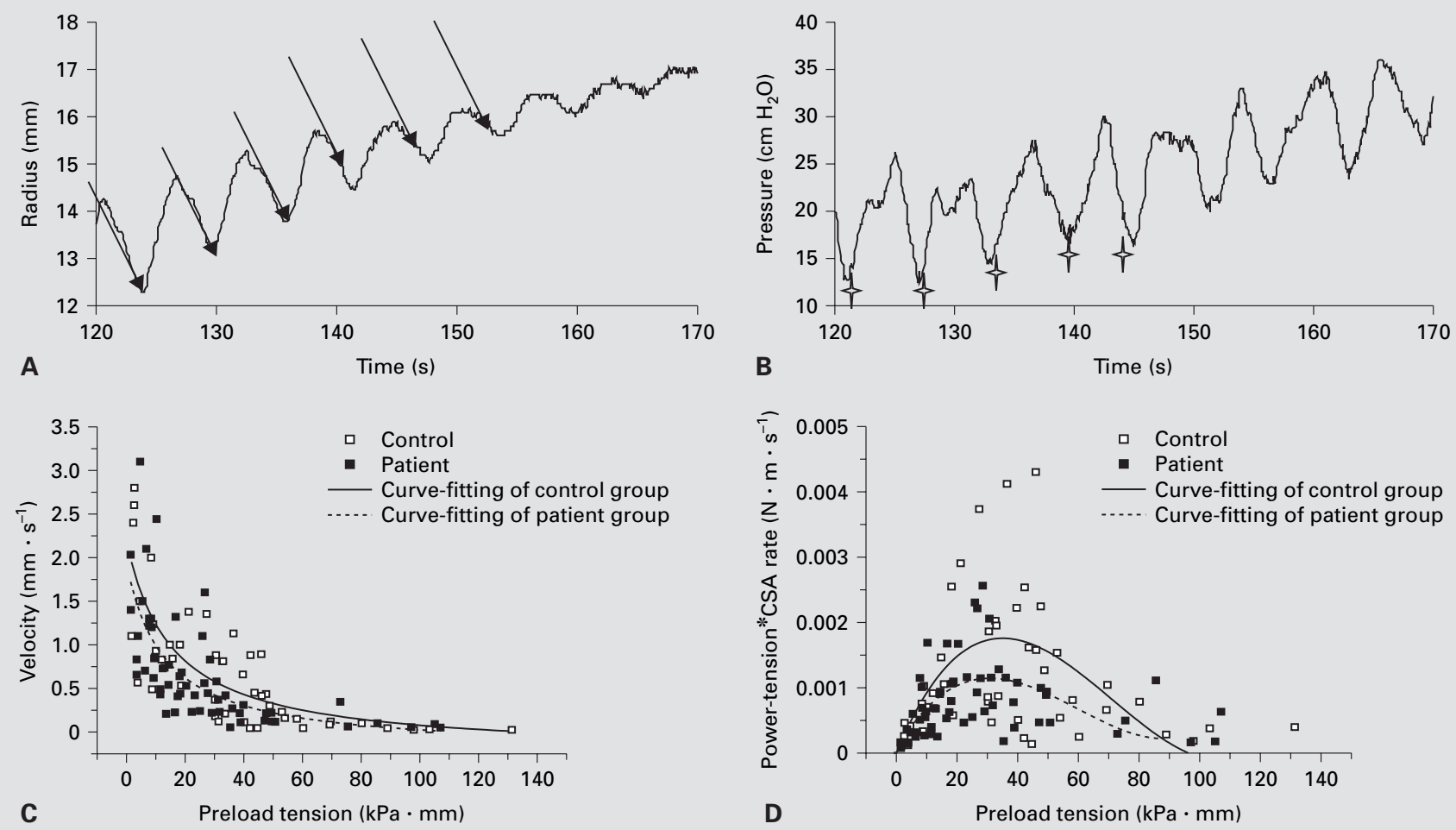

Fig. 7. A Arrows indicate that the slope (velocity) decreases at high loads. B The stars indicate the preload tension. Force-velocity curves in $\mathbf{C}$ and force-power curves in D are represented as circumferential preload tensionradius shortening velocity and preload tension-circumferential preload tension*CSA rate in SS patients (dashed lines) and controls (solid lines). The tension-velocity data were curve-fitted using Hill's equation and the power-tension data using a cubic polynomial function.

Fig. 8. The probe assembly for multimodal

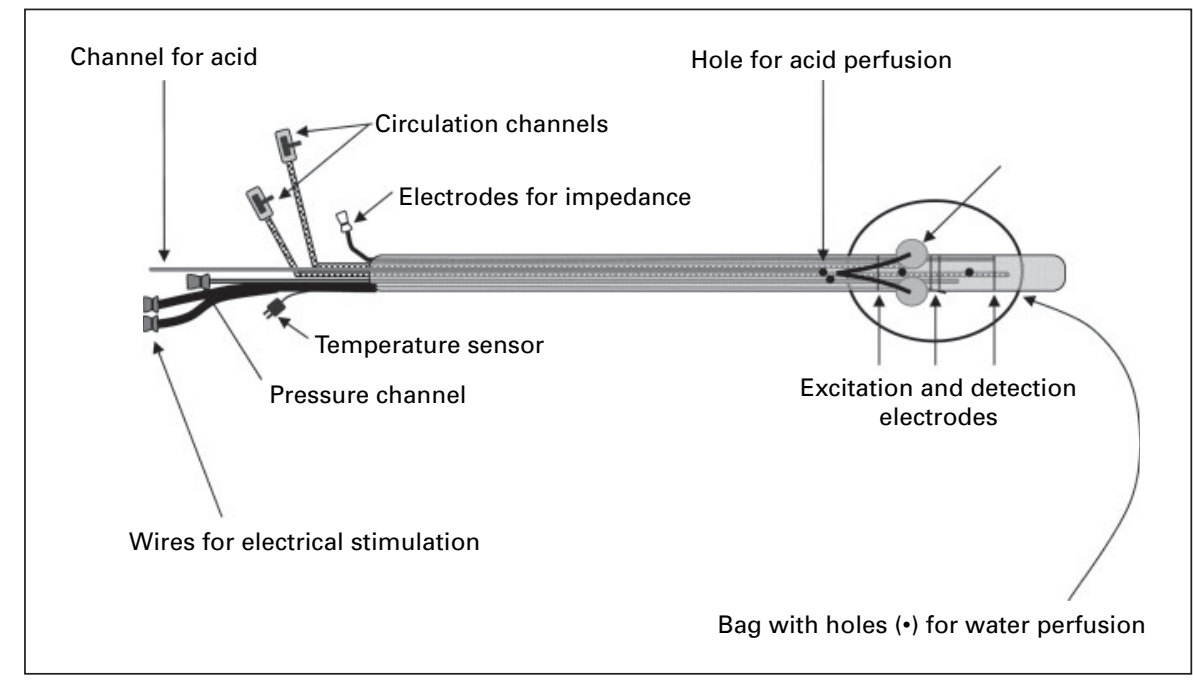




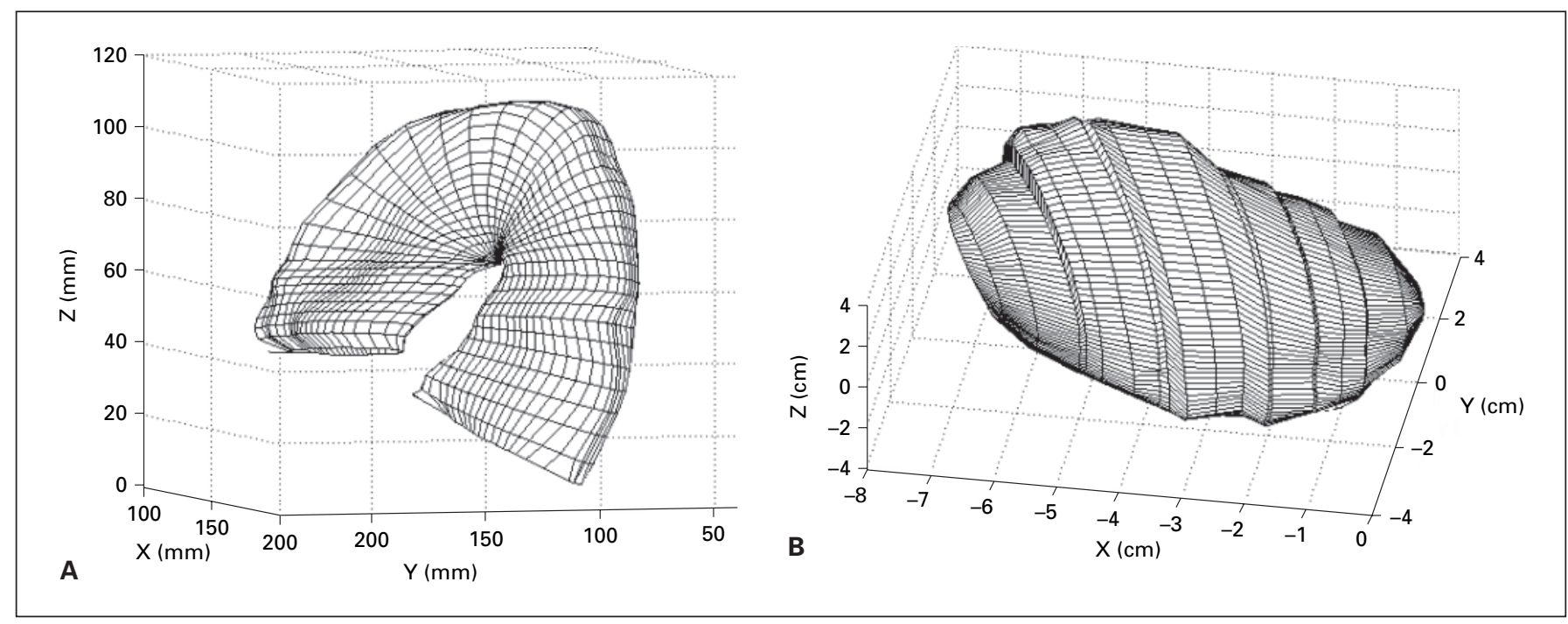

Fig. 9. 3D sigmoid colon (A) and 3D antrum (B) configuration reconstructed from the MRI scanning images and $3 \mathrm{D}$ ultrasound system.

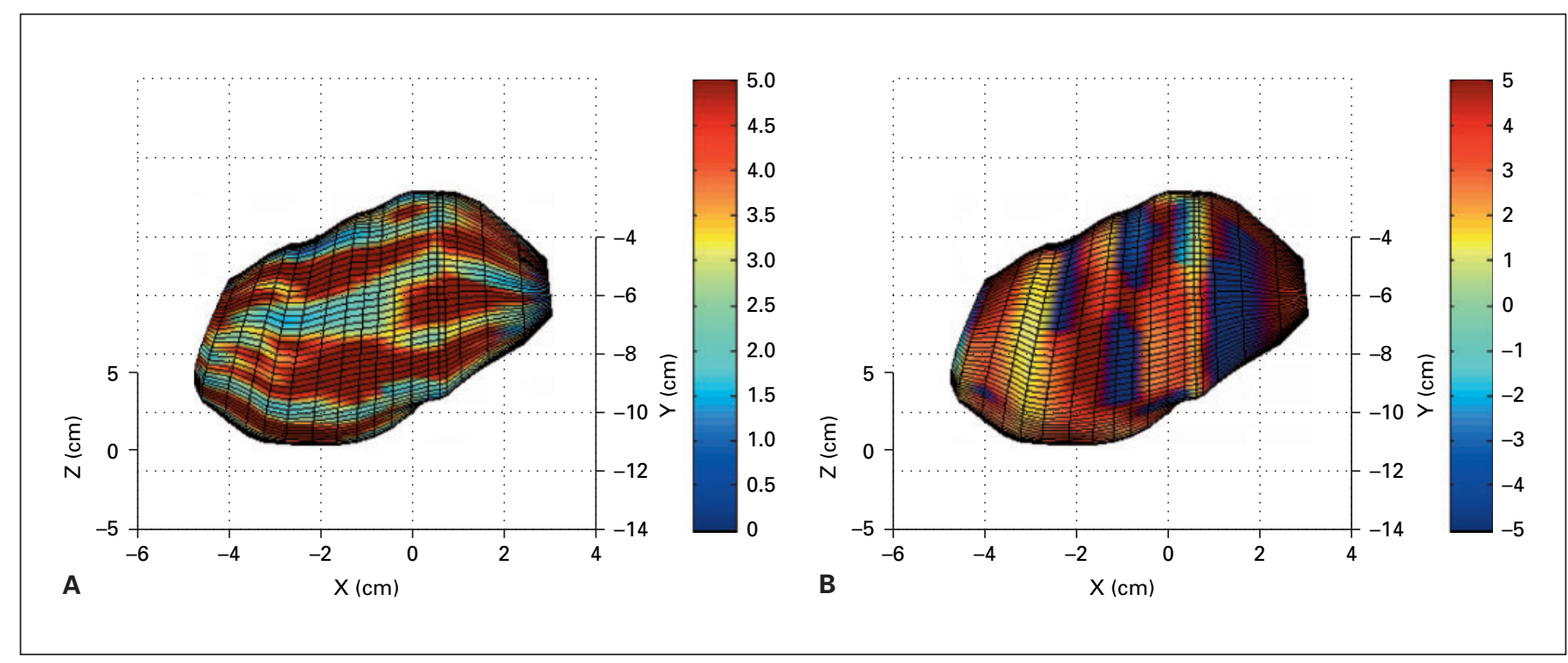

Fig. 10. The circumferential (A) and the longitudinal (B) radii of curvature distribution on a $3 \mathrm{D}$ antrum surface. The color change from blue to red represents the increase of the radii.

aids of medical imaging technology, the 3D geometry of the complex organ during balloon distension can be reconstructed from a series of images. Recently, the 3D antrum [5], stomach [41], and rectum [6] at several distension volumes were reconstructed from 3D ultrasound and MRI scanning images (fig. 9). It is clear from these $3 \mathrm{D}$ reconstructions that the assumptions in previous pressure-volume studies were not fulfilled. The surface data in the 3D analysis were used to compute the principal radii of curvature to be used in further biomechanical analysis.

The radii of curvature analysis in the antrum show a large variation which indicates that tension estimations based on Laplace's law is dubious (fig. 10). Several previ- 
ous studies found that the sensory data correlated to the tension $[9,42]$. The 3D tension distributions show that it has little meaning to compute an average tension. If the radii of curvatures obtained are analyzed using Laplace's law for a thin-walled structure, a very heterogeneous distribution of tensions is obtained. The computation of tension in such complex geometry requires numerical methods such as finite element analysis. Furthermore, it may be more meaningful to know the peak tension rather than an average tension when evaluating distension-sensitive sensory responses. For instance, the previous 3D stomachs study indicates that the peak tension occurs in the gastric body at the anterior surface. If a correlation exists between tension and symptoms from the stomach, this suggests that the location of the peak stress has a different sensitivity to stimuli than other parts of the stomach. Another pathophysiological question related to biomechanical analysis is to estimate the most likely location of rupture in the GI organs. The rupture or mechanical damage will occur at points where the peak stress exceeds the di- minishing failure strength of the distended wall. The wall stress can be estimated from the tension and the wall thickness.

\section{Conclusion}

Balloon-distension techniques have been used for a long time in experimental and clinical studies of the GI tract. They have added significantly to our knowledge on GI physiology and pathophysiology. Currently, new advanced techniques are being developed. These are especially useful for obtaining the geometric structure of the organ under study and for evaluation of the muscle function in a mechanical sense. Important data can be obtained on the viscoelastic properties of the GI tract and on the mechanosensory mechanisms involved in pain. Consequently, balloon distension will continue to be useful in future research and clinical work.

\section{References}

-1 Rao SS, Gregersen H, Hayek B, Summers RW, Christensen J: Unexplained chest pain: the hypersensitive, hyperreactive, and poorly compliant esophagus. Ann Intern Med 1996;124: 950-958.

-2 Schoenhagen P, Ziada KM, Vince DG, Nissen SE, Tuzcu EM: Arterial remodeling and coronary artery disease: the concept of 'dilated' versus 'obstructive' coronary atherosclerosis. J Am Coll Cardiol 2001;38:297-306.

-3 Shafik A: Esophago-sphincter inhibitory reflex: role in the deglutition mechanism and esophageal achalasia. J Invest Surg 1996;9:37-43.

4 Liao D, Zhao J, Gregersen H: Three-dimensional geometry analysis of the stomach in type II diabetic GK rats. Diabetes Res Clin Pract 2006;71:1-13.

-5 Liao D, Gregersen H, Hausken T, Gilja OH, Mundt M, Kassab G: Analysis of surface geometry of the human stomach using real-time 3-D ultrasonography in vivo. Neurogastroenterol Motil 2004; 16:315-324.

-6 Frokjaer JB, Liao D, Bergmann A, et al: Threedimensional biomechanical properties of the human rectum evaluated with magnetic resonance imaging. Neurogastroenterol Motil 2005; 17:531-540.

7 Phillips RJ, Powley TL: Tension and stretch receptors in gastrointestinal smooth muscle: re-evaluating vagal mechanoreceptor electrophysiology. Brain Res Brain Res Rev 2000;34: $1-26$.

Balloon-Distension Studies in the Gastrointestinal Tract: Current Role
8 Whitehead WE, Delvaux M: Standardization of barostat procedures for testing smooth muscle tone and sensory thresholds in the gastrointestinal tract. The Working Team of GlaxoWellcome Research, UK. Dig Dis Sci 1997;42: 223-241.

9 Azpiroz F, Malagelada JR: Physiological variations in canine gastric tone measured by an electronic barostat. Am J Physiol 1985;248: G229-G237.

10 Distrutti E, Azpiroz F, Soldevilla A, Malagelada JR: Gastric wall tension determines perception of gastric distention. Gastroenterology 1999;116:1035-1042.

11 Van der Schaar PJ, Lamers CB, Masclee AA: The role of the barostat in human research and clinical practice. Scand J Gastroenterol Suppl 1999;230:52-63.

12 Camilleri M: Testing the sensitivity hypothesis in practice: tools and methods, assumptions and pitfalls. Gut 2002;51(suppl 1):i34-i40.

13 Fass R, Naliboff B, Higa L, et al: Differential effect of long-term esophageal acid exposure on mechanosensitivity and chemosensitivity in humans. Gastroenterology 1998;115:13631373.

14 Khan MI, Read NW, Grundy D: Effect of varying the rate and pattern of gastric distension on its sensory perception and motor activity. Am J Physiol 1993;264:G824-G827.

15 Gregersen H, Barlow J, Thompson D: Development of a computer-controlled tensiometer for real-time measurements of tension in tubular organs. Neurogastroenterol Motil 1999;11: 109-118.
16 Gregersen H, Gilja OH, Hausken T, et al: Mechanical properties in the human gastric antrum using B-mode ultrasonography and antral distension. Am J Physiol 2002;283:G368G375.

17 Gregersen H, Jensen LS, Djurhuus JC: Changes in oesophageal wall biomechanics after portal vein banding and variceal sclerotherapy measured by a new technique. An experimental study in rabbits. Gut 1988;29:1699-1704.

18 Pedersen J, Reddy H, Funch-Jensen P, ArendtNielsen L, Gregersen H, Drewes AM: Differences between male and female responses to painful thermal and mechanical stimulation of the human esophagus. Dig Dis Sci 2004;49: 1065-1074

19 Gao C, Petersen P, Liu W, Arendt-Nielsen L, Drewes AM, Gregersen H: Sensory-motor responses to volume-controlled duodenal distension. Neurogastroenterol Motil 2002;14:365374.

20 Drewes AM, Schipper KP, Dimcevski G, et al: Multimodal assessment of pain in the esophagus: a new experimental model. Am J Physiol 2002;283:G95-G103.

21 Drewes AM, Schipper KP, Dimcevski G, et al Multi-modal induction and assessment of allodynia and hyperalgesia in the human oesophagus. Eur J Pain 2003; 7:539-549.

22 Pedersen J, Gao C, Egekvist H, et al: Pain and biomechanical responses to distention of the duodenum in patients with systemic sclerosis. Gastroenterology 2003;124:1230-1239. 
-23 Gregersen H, Christensen LL: Pressure-crosssectional area relations and elasticity in the rabbit oesophagus in vivo. Digestion 1996;57: 174-179.

-24 Drewes AM, Gregersen H, Arendt-Nielsen L: Experimental pain in gastroenterology: a reappraisal of human studies. Scand J Gastroenterol 2003;38:1115-1130.

-25 Drewes AM, Babenko L, Birket-Smith L, Funch-Jensen P, Arendt-Nielsen L: Induction of non-painful and painful intestinal sensations by hypertonic saline: a new human experimental model. Eur J Pain 2003; 7:81-91.

26 Gao C, Arendt-Nielsen L, Liu W, Petersen P, Drewes AM, Gregersen H: Sensory and biomechanical responses to ramp-controlled distension of the human duodenum. Am J Physiol 2003;284:G461-G471.

-27 Drewes AM, Gregersen H, Arendt-Nielsen L: Experimental pain in gastroenterology: a reappraisal of human studies. Scand J Gastroenterol 2003;38:1115-1130.

-28 Petersen P, Gao C, Arendt-Nielsen L, Gregersen H, Drewes AM: Pain intensity and biomechanical responses during ramp-controlled distension of the human rectum. Dig Dis Sci 2003;48:1310-1316.
29 Drewes AM, Pedersen J, Liu W, ArendtNielsen L, Gregersen H: Controlled mechanical distension of the human oesophagus: sensory and biomechanical findings. Scand $\mathbf{J}$ Gastroenterol 2003;38:27-35.

30 Andersen IS, Gregersen H, Buntzen S, Djurhuus JC, Laurberg S: New probe for the measurement of dynamic changes in the rectum. Neurogastroenterol Motil 2004;16:99105.

31 McMahon BP, Frokaer JB, Drewes AM, Gregersen H: A new measurement of oesophagogastric junction competence. Neurogastroenterol Motil 2004; 16:543-546.

32 Mayrand S, Diamant NE: Measurement of human esophageal tone in vivo. Gastroenterology 1993; 105:1411-1420.

33 Zhang X, Tack J, Janssens J, Sifrim DA: Neural regulation of tone in the oesophageal body: in vivo barostat assessment of volume-pressure relationships in the feline oesophagus. Neurogastroenterol Motil 2004;16:13-21.

34 Gregersen H: Biomechanics of the gastrointestinal tract. London, Springer, 2002.
35 Gregersen H, Kassab G: Biomechanics of the gastrointestinal tract. Neurogastroenterol Motil 1996;8:277-297.

36 Tøttrup A, Forman A, Uldbjerg N: Mechanical properties of isolated human esophageal smooth muscle. Am J Physiol 1990;256:338343.

37 Hill AV: The mechanics of active muscle. Proc R Soc Lond B Biol Sci 1953;141:104-117.

38 Guyton AC, Hall GE: Textbook of Medical Physiology. Philadelphia, Saunders, 2002.

39 Komi P: Neuromuscular performance: factors influencing force and speed production. Scand J Sports Sci 1979;1:2-15.

40 Staahl C, Drewes AM: Experimental human pain models: a review of standardised methods for preclinical testing of analgesics. Basic Clin Pharmacol Toxicol 2004;95:97-111.

41 Liao D, Zhao J, Gregersen H: Regional surface geometry of the rat stomach based on threedimensional curvature analysis. Phys Med Biol 2005;50:231-246.

42 Azpiroz F, Malagelada JR: Gastric tone measured by an electronic barostat in health and postsurgical gastroparesis. Gastroenterology 1987;92:934-943. 